\title{
Simulation of Feedback-driven PCR Assays on a 2D Electrowetting Array using a Domain-Specific High-level Biological Programming Language
}

\author{
Christopher Curtis Philip Brisk \\ Department of Computer Science and Engineering \\ University of California, Riverside \\ Riverside, CA 92521 \\ ccurt002@ucr.edu, philip@cs.ucr.edu
}

\begin{abstract}
The motivation of our work is to demonstrate that scientists can describe biochemical assays using a high-level domain-specific programming language and can execute them automatically on a two-dimensional electrowetting array featuring integrated sensors that provide feedback in real-time to the host PC that controls the integrated system. The fundamental research problem that this paper addresses is how to express, at a high level of abstraction, the acquisition of sensory information from the device, and the computation on that data, which is required to perform real-time decision making in response. The approach that we have taken is first, to create a new version of BioCoder, a programming language for automated biology, which we have specialized for elecrowetting arrays featuring integrated sensors, and second, to use this language to specify two feedback-driven Polymerase Chain Reaction (PCR) assays at the desired level of abstraction. Our methodology is to evaluate the performance of these assays using a custom-build runtime system to control the execution of feedback-driven assays on a cycle-accurate software simulator that accurately characterizes the behavior of the electrowetting platform during assay execution. The result of this experiment is successful simulation of these non-trivial feedback-driven assays, starting from a high-level specification, which allows us to conclude that high-level programming language design and implementation targeting electrowetting (or other competing laboratory-on-a-chip technologies) is feasible.
\end{abstract}

Keywords: Digital Microfluidics, Cyber-physical Integration, Real-time Decision-making, Polymerase Chain Reaction (PCR)

\section{INTRODUCTION}

Electrowetting-on-Dielectric (EWoD) has enabled the creation of software-programmable digital microfluidic platforms, which can perform a wide variety of biochemical applications $[6,10,20$, 21, 23] (Fig. 1). By applying a voltage sequence to a two dimensional array of electrodes (Fig. 2), basic droplet operations such as dispensing, mixing, dilution, transport, splitting, merging, and storage can be combined in a spatially parallel manner to execute macro-scale biochemical reactions on a micro-scale device (Fig. 3). Recent advances in sensor integration [4, 8, 16, $17,22,25-27,30]$ provide the ability to execute intelligent computer-controlled assays that obtain feedback and make decisions in real-time based on sensor readings (Fig. 4).

Compared to other microfluidic technologies, such as large scale integration of pneumatically controlled microvalves [19], DMFBs offer three key advantages. Firstly, there are no moving mechanical components-droplet motion is induced through electrostatic actuation. Secondly, control is provided exclusively through electricity, which eliminates the need for external pressure sources, which significant inhibit the ability to remove other LoCs from the laboratory for usage in the field. Thirdly, the electrical interface provided by a DMFB is a natural match for computer control; thus, there has been interest in the development of programming languages, compiler technology and software control of DMFBs in recent years [5, 9, 11, 14, 18, 27, 28].

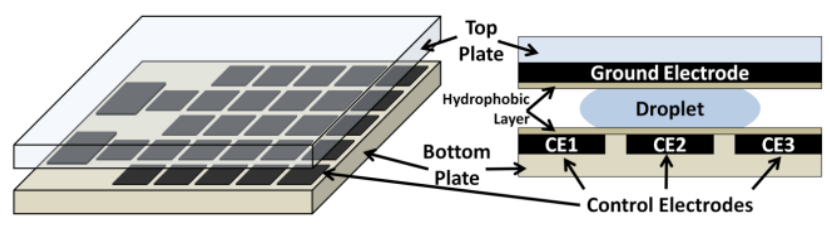

Figure 1. Left: Depiction of a two-dimensional EWoD digital microfluidic platform; Right: one-dimensional cross-section.

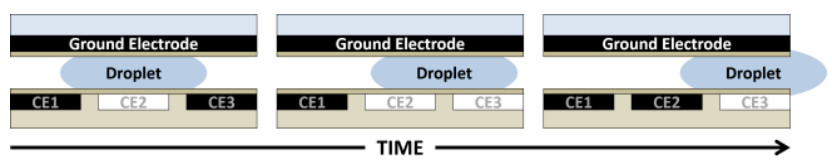

Figure 2. Depiction of electrostatic droplet actuation; white control electrodes are activated (voltage applied); black control electrodes are deactivated (no voltage applied).
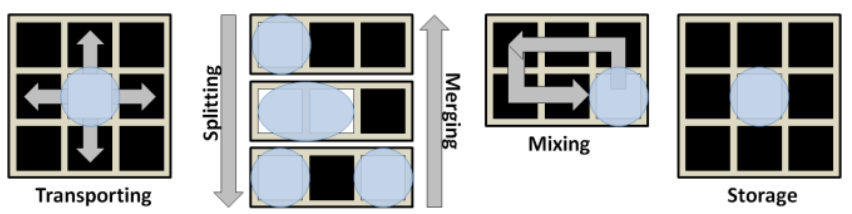

Figure 3. The five basic operations supported by a digital microfluidic system.

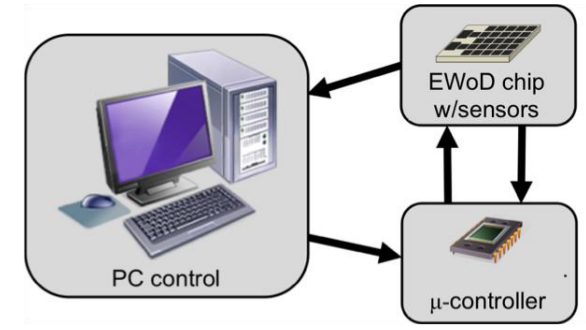

Figure 4. A feedback-driven digital microfluidic platform. 
Historically, users of digital microfluidic platforms programmed the electrode sequences by hand, a tedious, time-consuming, and error-prone process. Manual programming does not readily adapt to the sensor-driven real-time feedback control situation (Fig. 4), where feedback provided from the device to the host PC controller cannot be predicted a-priori. This context necessitates the specification of a feedback-driven assay using a domain-specific programming language, coupled with the software infrastructure (compiler and runtime system) that coordinates the entire system [11]. Such a system offers a potential productivity boon to users of digital microfluidic systems; however, it requires them to think about their research at a higher level of abstraction, akin to professional software development, as opposed to biochemistry.

We report the specification and simulated execution of two feedback-driven assays using a domain-specific language and software infrastructure developed previously by the authors [11]. The assays that we simulate are PCR variants: one uses sensory feedback to terminate thermocycling early when the initial droplet contains insufficient product for amplificiation [18]; the other uses integrated sensors to detect droplet evaporation during thermocycling in an air-matrix digital microfluidic system, and performs just-in-time replenishment using droplets of solvent to ensure that the reaction volume remains within an acceptable window [14]. This study establishes the viability and validity of software-driven programming of digital microfluidic EWoD systems, and serves to motivate further research on this topic.

\section{BIOCODER: A HIGH-LEVEL PROGRAMMING LANGUAGE FOR DIGITAL MICROFLUIDICS}

The assays are specified using BioCoder, a somewhat more general language (technically, a C++ library) originally developed at Microsoft Research, India [3], and subsequently specialized by the authors for digital microfluidics [11]. The authors introduced extensions to BioCoder obtain sensory feedback from the EWoD device, and to take actions (conditional statements; loops) based on computations performed on the sensor readings. The version of BioCoder used in this paper is essentially the same as in Ref. [11].

Unlike traditional programming languages for computing, BioCoder naturally supports fluidic types and fluidic operations,. Fluidic types enable the programmer to declare program variables to represent fluids; fluidic operations enable the programmer to specify high-level operations that operate on fluids (e.g., mix, split) without worrying about low-level implementation issues, including: (1) selecting a location on-chip to perform the operation; (2) transporting droplets to/from the operation; (3) the electrode activation sequence required to execute the operation; and (4) coordinating the parallel execution on multiple operations.

Many BioCoder operations are timed (e.g., mix fluids A and B for 10 seconds), which is different than traditional programming languages (e.g., integer or floating-point addition in $\mathrm{C} / \mathrm{C}++$ ). Fluids also possess intrinsic properties, such as temperature and volumes, which need to be monitored during assay execution; a variable in a traditional programming language may take a discrete range of values, but possess no additional inherent information beyond its value at a given point in time.

Although these features are specific to BioCoder at present, the authors anticipate that they are inherent to any biologicallyoriented domain-specific programming language that could potentially emerges in the future as well. For a description of the BioCoder syntax and execution model, please refer to Ref. [11].

\section{BIOCODER PCR APPLICATIONS}

\subsection{The Polymerase Chain Reaction (PCR)}

The Polymerase Chain Reaction (PCR) is a technique used in molecular biology to amplify a relatively small quantity of a DNA sequence (as little as a single strand) by several orders of magnitude. Applications of PCR include DNA cloning, genetic analysis, diagnosis of hereditary diseases, DNA-based forensics and paternity testing, and diagnosis of infectious diseases.

PCR relies on thermal cycling, i.e., repeatedly heating and cooling the reaction to melt and replicate the DNA, through a defined series of temperature steps. PCR employs primers (short DNA fragments) that contain sequences that are complementary to the target region, along with a heat-stable DNA polymerase consisting of enzymes that construct DNA molecules from an assemblage of nucleotides.

As an example, the PCR phase of an influenza detection assay [23] proceeds as follows:

1. Measure out $240 \mu \mathrm{l}$ of PCR reagent s

2. Add $600 \mu$ of DNA

3. Vortex the mixture for 1 second

4. Incubate the mixture at $92^{\circ} \mathrm{C}$ for 30 seconds

5. For $\mathrm{i}=1$ to $35 / /$ Thermocycling loop

6. Incubate the mixture at $92^{\circ} \mathrm{C}$ for 5,000 seconds

7. Incubate the mixture at $55^{\circ} \mathrm{C}$ for 10,000 seconds

8. Incubate the mixture at $72^{\circ} \mathrm{C}$ for 20,000 seconds

9. $\quad$ End For

10. Incubate the mixture at $72^{\circ} \mathrm{C}$ for 60,000 seconds

11. [Subsequent influenza detection assay steps...]

The thermocycling process shown in the example above does not rely on sensory feedback to monitor the state of the DNA product being amplified. The next two subsections describe enhancements to the basic PCR thermocycling protocol that leverage sensors integrated into a digital microfluidic chip to optimize the process.

\subsection{Statistical DNA Amplification}

The number of DNA strands in a dispensed droplet in a digital microfluidic chip has been shown to be a random variable that follows a Poisson distribution when the density of DNA strands in a sample is low $[15,33]$. PCR will fail when applied to a so-called "empty" droplet, which has an insufficient number of initial DNA strands [32]. Feedback from integrated sensors (fluorescence signals) during the early stages of thermocycling can predict whether or not a droplet is empty [7, 13]. Droplets that are predicted to be empty are discarded early, saving time that would otherwise be wasted on an unsuccessful amplification attempt. Using this model, the thermocycling proceeds as follows:

1. Warmup phase (traditional thermocycling for a limited time).

2. Detection phase (traditional thermocycling with a DNA fluorescence detection measurement taken after each cycle).

3. Go/No-Go decision: based on the sequence of fluorescence signals, the droplet is categorized as "empty" or "non-empty." If the droplet is categorized as "empty," thermocycling terminates preemptively; otherwise, thermocycling continues.

4. If the droplet is not discarded, then thermocycling continues and PCR completes as expected.

BioCoder source code for the probabilistic PCR algorithm is included as supplementary information. 
Probability Model: The number of DNA strands in a droplet grows exponentially when PCR amplification is successful. The probability model developed by Luo et al. [18], which we adopt, assumes this exponential growth trend and introduces Eq. (1), below to calculate the confidence percentage $p^{i}$ at cycle $i$. The confidence percentage indicates the probability of whether a droplet will exhibit detectable DNA at that cycle. The terms $N$ and $\mathrm{p}^{*}$ respectively represent the minimum number of thermocycles required to achieve detection, and a scaling factor that are unique to the DNA sample that is being amplified by PCR; thus, this model requires empirical calibration before it can be used.

$$
p^{i}=\left\{\begin{array}{cc}
0, & \text { if } i<N \\
p^{*} * 2^{i-N}, & \text { if } N \leq i \leq N-\log _{2} p^{*} \\
1, & \text { if } N-\log _{2} p^{*}<i
\end{array}\right.
$$

Once the confidence value is calculated, Eqs. (2) and (3), below are used to calculate $P(G)$, the probability of a good (amplifiable) droplet, or $P\left(G^{C}\right)$, the probability of a bad (empty) droplet, given that no DNA was observed at cycle $k$. Based on these equations, one can determine the minimum number of thermocycles needed to allow DNA amplification to the point of detection.

$$
\begin{aligned}
& P\left(G \mid A_{k}\right)=\frac{\prod_{i=1}^{k}\left(1-p_{i}\right) * P(G)}{\Pi_{i=1}^{k}\left(1-p_{i}\right) * P(G)+1-P(G)} \\
& P\left(G^{c} \mid A_{k}\right)=\frac{1-P(G)}{\Pi_{i=1}^{k}\left(1-p_{i}\right) * P(G)+1-P(G)}
\end{aligned}
$$

If DNA is detected at any cycle $k^{*} \leq k$, then the thermocycling runs to completion; if no DNA is detected during the $k^{\text {th }}$ thermocycle, then the droplet is discarded.

\subsection{Real-time Solvent Replenishment}

Fig. 1 illustrates a closed digital microfluidic system with a top plate; in practice, many application environments necessitate open digital microfluidic platforms in which droplets are surrounded by air. In these platforms, droplet evaporation occurs naturally, especially when droplets are heated, as is the case in PCR thermocycling. Enzymatic reactions, for example, are sensitive to variations in reactant concentration. To address this concern, Jebrail et al. [14] implemented a digital microfluidic system that pre-heats droplets of solvent and uses them to replenish an otherwise perpetually diminishing reaction volume during PCR thermocycling. Replenishment occurs when a droplet has lost 15$20 \%$ of its volume. Pre-heating the replenishment droplets reduces the impact of temperature changes during replenishment.

As noted by the authors, volume loss was determined by visual inspection and replenishment was accomplished through manual intervention by the user. In principle, droplet volumes can be accurately estimated using real-time computer vision algorithms [5, 9, 28], capacitive sensors [16], and/or impedance sensors [25]. Our work, albeit using simulation, describes how this process can be automated through programmability, and should be compatible with any droplet volume estimation technology and/or algorithms.

We implemented two versions of PCR with droplet replenishment. The static version replenishes once during each thermocycle; since the decision to replenish is always affirmative, this version does not detect the volume of the droplet undergoing thermocycling. The dynamic version performs detection, and only replenishes when the volume of the droplet undergoing thermocycling falls beneath a tolerable threshold.

BioCoder source code for the static/dynamic versions of PCR with solvent replenishment are included as supplementary information.

\section{SIMULATOR}

We use a software-based digital microfluidic simulator whose design and implementation is described in Ref. [11]. Our compiler converts the BioCoder application into a bytecode format that is fed into a real-time interpreter, which communicates with the simulator to execute the simulated assay.

\subsection{Bytecode Format}

The bytecode format represents the assay as a control flow graph (CFG), a standard program representation used by programming languages and compilers dating back to 1970 (if not earlier) [1]. Each CFG node is called a basic block, and represents a maximum-length sequence of operations without any branches or branch targets interleaved. The CFG has dedicated entry/exit nodes, which represent the beginning/termination of the program. Internal nodes may have one or more successors; each internal node with multiple successors ends with a conditional operation (e.g., an if-then-else statement, loop branch condition, etc.).

While generating the ByteCode, the compiler performs a localized dependence analysis on each basic block, converted it into a Directed Acyclic Graph (DAG) called a Data Flow Graph (DFG). This enables the interpreter (described below) to identify operations that can be scheduled concurrently in order to exploit the spatial parallelism provided by the digital microfluidic system.

In the context of a feedback-driven BioCoder application, the conditional operations are the results of a computation performed on sensory data obtained from the digital microfluidic chip. Thus, in the most general case, a basic block will typically consist of a sequence of fluidic operations followed by reading one or more integrated sensors (this generalizes to external sensors, real-time video monitoring equipment, etc.), computation performed on the sensory data, and then execution of a conditional branch based on the outcome of the computation.

For example, in the case of real-time solvent replenishment, the conditional outcome would be: (1) continue thermocycling without replenishment; (2) replenish the droplet, then continue thermocycling; or (3) terminate (i.e., a sufficient number of thermocycles have been performed, and the next phase of the assay can proceed with a droplet that presumably contains an ample supply of amplified product.

\subsection{Execution Model}

Fig. 5 illustrates the execution model employed by the interpreter. The input is a representation of the assay in the CFG-based bytecode format described above. Starting with the CFG entry node, the interpreter executes each basic block. If basic block $b_{1}$ has a single successor $b_{2}$, then the interpreter executes $b_{2}$ next. If there are multiple successors, $b_{2}, \ldots, b_{k}$, then the interpreter selects the next one to execute based on the result of the conditional operation at the end of $b_{1}$. Execution terminates once the CFG exit node executes.

As shown in Fig. 5, each basic block is represented as a DAG. To execute the basic block, the interpreter schedules the operations, places them in the 2-dimensional digital microfluidic chip surface, and computes droplet routes between operations in real-time; computation speed is important, because the assay is temporarily paused while the interpreter figures out how to execute the DAG. After scheduling, placing, and routing the DAG, the interpreter executes the operations, processes sensory feedback, and selects the next CFG node to execute, as described above. 


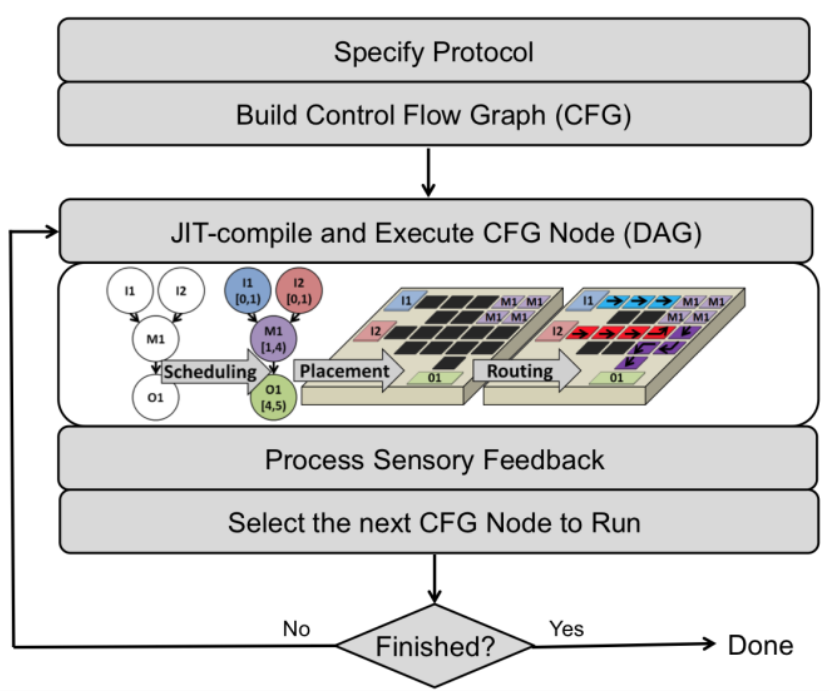

Figure 5. Execution model for the BioCoder simulator.

\subsection{Virtual Topology}

The simulator employs a virtual topology [11], illustrated below in Fig. 6. The virtual topology is an abstraction layer, that simplifies the scheduling, placement, and routing process, as described above. I/O ports on the perimeter of the digital microfluidic chip are not shown. The virtual topology divides the chip into four work modules (blue), which perform assay operations (mixing, dilution, etc.). When not performing an operation, each work module can store up to 4 droplets on-chip for later use. Each work module also has an external device attached: two have heaters (flames; $98^{\circ}$ and $68^{\circ}$ respectively); one has a cooler (snowflake; $50^{\circ}$ ); and one has a detector (magnifying glass). Thus, each work module can perform one enhanced operation in addition to the basic assay operations. The cells between the work modules form a network of unidirectional city streets with traffic circles at intersections; this network of streets is used to transport droplets between work modules I/O ports on the perimeter of the chip.

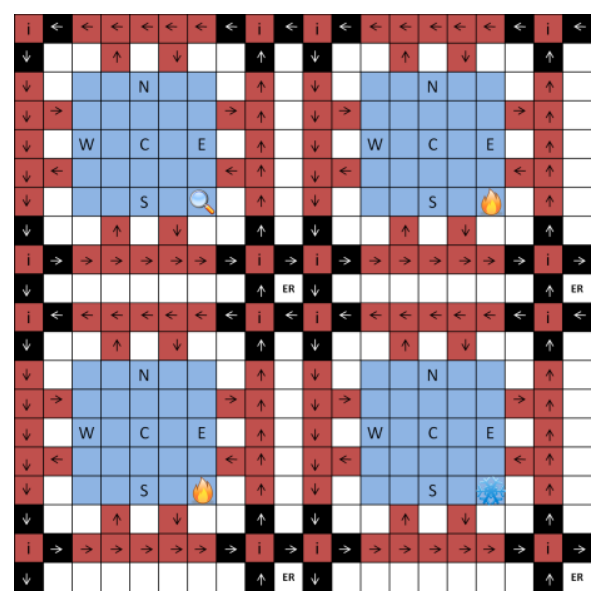

Figure 6. Illustration of the simulator's virtual topology.
The work module abstraction simplifies the algorithmic design of the scheduler and placer. The scheduler knows that it can issue up to four operations in parallel, with limitations based on the external devices attached to each work module (e.g., it is not possibly to perform two detection operations at the same time). For each scheduled operation at a given time step, the placer has a very simple decision to make: which work module to choose for the operation. Specialized operations using external devices get the highest priority for placement; other assay operations that have fewer restrictions are placed afterward. The router, meanwhile, uses the network of city streets to transport droplets. Prior work has shown that this traffic network enables the usage of straightforward and provably deadlock-free droplet routing algorithms [11], which reduces the router's computational burden.

This virtual topology abstraction is not an absolute requirement to use BioCoder to execute an assay on a digital microfluidic chip; it is simply an implementation choice that was made at the time the simulator was developed in order to simplify the algorithmic requirements for real-time scheduling, placement, and routing. Although our simulation results reflect our decision to use the virtual topology, similar results could be obtained for a system using any algorithm(s) for scheduling, placement, and routing.

\section{SIMULATION RESULTS}

\subsection{Probabilistic PCR}

When the probabilistic model suggests that the droplet undergoing amplification is good, the total execution time of PCR is 1811.25 seconds; if the droplet is empty, then amplification terminates preemptively after 908.87 seconds. For a single empty droplet, the probabilistic model provides a savings of 902.38 seconds $(49.8 \%)$ in terms of execution time. These estimates include the time required for droplet routing in addition to the time spent executing assay operations (heating, detection, etc.)

We used the simulator to vary $P(G)$, the probability that a given droplet is good. For each probability value we considered, we ran ten tests. Table I reports the number of good and empty droplets, along with the savings accrued by preemptively terminating amplification when a droplet is predicted to be empty. As one would expect, the greatest savings are achieved for low values of $P(G)$, where a larger number of droplets are empty, and therefore benefit from early termination.

\subsection{PCR with Solvent Replenishment}

Using pseudo-random number generation in the BioCoder source code, we vary the per-thermocycle probability that replenishment is required, which is a proxy for the evaporation rate. For a $100 \%$ replenishment probability, we use the static algorithm, which omits volume detection during each thermocycle; for replenishment probabilities lower than $100 \%$, we use the dynamic algorithm, which performs volume detection each thermocycle. For each replenishment probability, we run the algorithm using five random number seeds and report the average execution time across the five runs. To reduce simulation time, we execute 9 thermocycles, as opposed to the 33 thermocycles suggested by the authors of the replenishment study [14].

Table II reports the results of this experiment. Dynamic replenishment yields the shortest overall execution times because it does not incur the overhead of droplet volume detection. When droplet replenishment occurs, overhead is incurred due to dispensing, heating, and routing the replenishment droplet. In the dynamic case, faster execution times were achieved for lower replenishment probabilities, meaning that fewer thermocycles incur the replenishment overhead. 
Table I. Simulation results for the probabilistic PCR assay obtained while varying the probability that a given input droplet is "good" (i.e., contains a sufficient amount of initial DNA to achieve amplification).

\begin{tabular}{|c|c|c|c|}
\hline $\begin{array}{l}\text { Good Droplet } \\
\text { Probability } \\
\mathrm{P}(\mathrm{G})\end{array}$ & $\begin{array}{l}\text { Number of } \\
\text { Good } \\
\text { Droplets }\end{array}$ & $\begin{array}{l}\text { Number of } \\
\text { Empty } \\
\text { Droplets }\end{array}$ & $\begin{array}{l}\text { Probabilistic } \\
\text { Model Execution } \\
\text { Time Savings }\end{array}$ \\
\hline 0.25 & 1 & 9 & $44.8 \%$ \\
\hline 0.5 & 2 & 8 & $39.9 \%$ \\
\hline 0.65 & 5 & 5 & $24.9 \%$ \\
\hline 0.75 & 8 & 2 & $10.0 \%$ \\
\hline 0.85 & 9 & 1 & $5.0 \%$ \\
\hline
\end{tabular}

Table II. Simulation results for the probabilistic PCR assay obtained while varying the probability that a given input droplet is "good" (i.e., contains a sufficient amount of initial DNA to achieve amplification).

\begin{tabular}{lccccc} 
& Static & \multicolumn{4}{c}{ Dynamic } \\
\hline Replenishment Probability & $100 \%$ & $50 \%$ & $35 \%$ & $25 \%$ & $15 \%$ \\
\hline Execution Time (seconds) & 1217 & 1410 & 1361 & 1295 & 1238 \\
\hline
\end{tabular}

\section{RELATED WORK}

To the best of our knowledge, BioStream [31] developed at MIT, was the first high-level programming language for microfluidic technologies. The evaluation of BioStream was limited to dilution protocols, and the language and its compiler were never released publicly. According to private communication with the BioStream development team, it has been deprecated in favor of BioCoder.

The original intention behind BioCoder (as well as a competing language, EXACT [29]) was to improve scientific dissemination of biological protocols [3]. The output of the BioCoder compiler was a cookbook style, unambiguous, step-by-step Englishlanguage description of how to execute an assay, which could then be disseminated to the wider research community. The paper that introduced BioCoder suggested that it could be used to program a lab-on-a-chip, but they did not implement that feature.

Subsequently, Grissom et al. [11] specialized BioCoder to enable it to control a digital microfluidic chip and added conditional operations in order to support real-time decision-making based on sensory feedback; however, the use cases reported by Grissom et al. were simplistic and limited in scope. This paper provides two use cases, which are considerably more complex and practically usable by the wider research community, thereby providing a much stronger validation of the benefits of using high-level programming languages for digital microfluidics.

Another notable microfluidic programming language is the Aquacore Instruction Set (AIS), which is specific to the Aquacore architecture [2]. Aquacore is based on integrated microvalve technology, which manipulated continuous flows of fluid, rather than discrete droplets [19]. Aquacore is a collection of fluidic components that are connected to a centralized transport bus. The AIS includes operations to activate/deactivate/control each component, as needed, and to transfer fluids between components. Aquacore includes a high-level language that can be compiled more or less deterministically into AIS instructions. Aquacore and AIS share the same principle objectives as our work, but target a wholly different microfluidic technology.
Duke University reported software-driven EWoD cyber-physical integration in 2013 [12]. Capacitance sensors detect faults that occur when droplets become stuck due to defects and breakdowns that occur over time in the device substrate. The software updates pre-computed droplet routing paths in response, thereby ensuring correct assay operation, completely removing the human operator from the recovery process. In principle, this type of fault tolerance and recovery process could be integrated into the runtime system that controls an EWoD chip, such as ref. [11]. With respect to this work, it does not make sense to introduce fault mitigation into a high-level programming language targeting EWoD, because the user would be required to specify recovery procedures for each assay operation, which would complicate the programming task; moreover, this approach would be incomplete in situations where errors occur during the error recovery process.

\section{DISCUSSION AND CONCLUSION}

This paper has established the feasibility of programming nontrivial feedback-driven assays using high-level languages, and then executing them on digital microfluidic systems based on EWoD technology. Although the experimental results reported in this paper use a simulated EWoD platform, the transition to a realworld device is well within reach.

The long-term vision of our work is to empower the users of digital microfluidic systems to operate at a higher-level of abstraction in comparison to current practice. The biochemist of the future should evolve into a programmer, rather than a device expert. Specifying an assay using a high-level language allows the programmer to focus on the biochemistry that he/she wishes to perform, not the specifics of how to coax a complex and arguably counterintuitive device into doing the job; in other words, the compiler and interpreter eliminate the need for the user to worry about the device-level details and interfacing issues.

\section{ACKNOWLEDGMENTS}

This work was supported by the NSF under grant CNS-1035603. Any opinions, findings, and conclusions or recommendations expressed in this material are those of the authors and do not necessarily reflect those of the NSF.

\section{REFERENCES}

[1] F. E. Allen: Control flow analysis. ACM SIGPLAN Notices 5(7):1-19 (1970). DOI: http://dx.doi.org/10.1145/390013.808479

[2] A. Amin, M. Thottethodi, T. N. Vijaykumar, S. Wereley, and S. C. Jacobson: Aquacore: a programmable architecture for microfluidics. ISCA 2007: 254-265. DOI: http://dx.doi.org/10.1145/1250662.1250694

[3] V. Ananthanarayanan, and W. Thies: BioCoder: a programming language for standardizing and automating biology protocols. Journal of Biological Engineering 4(13): online. DOI: http://dx.doi.org/10.1186/1754-1611-4-13

[4] E. Barsoukov and J. R. Macdonald, Eds.: Impedence Spectroscopy: Theory, Experiments, and Applications. Wiley-Interscience (2005)

[5] A. S. Basu: Droplet morphometry and velocimetry (DMV): a video processing software for time-resolved, label-free tracking of droplet parameters. Lab on a Chip 13(10):18921901 (2013) DOI: http://dx.doi.org/10.1039/c3lc50074h

[6] Jean Berthier, Micro-Drops and Digital Microfluidics, Norwich, NY: William Andrew (2008) 
[7] D. Brassard, L. Malic, C. Miville-Godin, F. Normandin, and T. Veres: Advanced EWOD-based digital microfluidic system for multiplexed analysis of biomolecular interactions. IEEE MEMS 2011: 153-156. DOI: http://dx.doi.org/10.1109/MEMSYS.2011.5734384

[8] P. Cooreman, R. Thoelen, J. Manca, M. vandeVen, V. Vermeeren, L. Michiels, M. Ameloot, and P. Wagner: Impedimetric immunosensors based on the conjugated polymer PPV. Biosens. Bioelectron. 20(10):2151-6 (2005) DOI: http://dx.doi.org/10.1016/j.bios.2004.08.029

[9] R. Fobel, C. Fobel, and A. R. Wheeler: DropBot: an opensource, digital microfluidic control system with precise control of electrostatic driving force and instantaneous drop velocity measurement. Applied Physics Letters 102(10): 1935313 (2013) DOI: http://dx.doi.org/10.1063/1.4807118

[10] J. Gong, and C. J. Kim: Direct-referencing two-dimensionalarray digital microfluidics using multilayer printed circuit board. J. Microelectromech. S. 17(2):257-264 (2008) DOI: http://dx.doi.org/10.1109/JMEMS.2007.912698

[11] D. Grissom, C. Curtis, and P. Brisk: Interpreting assays with control flow on digital microfluidic biochips. ACM JETC 10(3): 24 (2014) DOI: http://dx.doi.org/10.1145/2567669

[12] K. Hu, B-N. Hsu, A. Madison, K. Chakrabarty, and R. B. Fair: Fault detection, real-time error recovery, and experimental demonstration for digital microfluidic biochips. DATE 2013: 559-564. DOI:

http://dx.doi.org/10.7873/DATE.2013.124

[13] D. Jary, A. Chollat-Namy, Y. Fouillet, J. Boutet, C. Chabrol, G. Castellan, D. Gasparutto, and C. Peponnet: DNA repair enzyme analysis on EWOD fluidic microprocessor. NSTINanotech, 2006: 2:554-557. Online: www.nsti.org/publications/Nanotech/2006/pdf/655.pdf

[14] M. J. Jebrail, R. F. Renzi, A. Sinha, J. Van De Vreugde, C. Gondhalekar, C. Ambriz, R. J. Meagher, and S. S. Branda: A solvent replenishment solution for managing evaporation of biochemical reactions in air-matrix digital microfluidics devices. Lab on a Chip 15:151-158 (2015) DOI: http://dx.doi.org/10.1039/c4lc00703d

[15] S. Koster, F. Angile, H. Duan, J. Agresti, A. Wintner, C. Schmitz, A. Rowat, C. Merten, D. Pisignano, A. Griffiths, and D. Weitz: Drop-based microfluidic devices for encapsulation of single cells. Lab on a Chip, 8(7):1110-1115 (2008) DOI: http://dx.doi.org/10.1039/b802941e

[16] Y. Li, H. Li, and R. J. Baker: Volume and concentration identification by using an electrowetting on dielectric device. IEEE DCAS 2014: 1-4. DOI: http://dx.doi.org/10.1109/DCAS.2014.6965350

[17] L. Luan, R. D. Evans, N. M. Jokerst, and R. B. Fair: Integrated optical sensor in a digital microfluidic platform. IEEE Sensors Journal 8(5):628-635 (2008) DOI: http://dx.doi.org/10.1109/JSEN.2008.918717

[18] Y. Luo, B. B. Bhattacharya, T-Y. Ho, and K. Chakrabarty: Design and optimization of a cyberphysical digitalmicrofluidic biochip for the polymerase chain reaction. IEEE Trans. on CAD of Integrated Circuits and Systems 34(1): 2942 (2015) DOI: http://dx.doi.org/10.1109/TCAD.2014.2363396

[19] J. Melin and S. R. Quake: Microfluidic large-scale integration: the evolution of design rules for biological automation. Annu. Rev. Biophys. Biomol. Struct. 36: 213231 (2007) DOI: $\underline{\text { http://dx.doi.org/10.1146/annurev.biophys.36.040306.13264 }}$ $\underline{6}$

[20] H. Moon, S. K. Cho, R. Garrell, and C. J. Kim: Low voltage electrowetting-on-dielectric. J. Appl. Phys. 92(7): 4080-4087 (2002) DOI: http://dx.doi.org/10.1063/1.1504171

[21] F. Mugele, and J. C. Baret: Electrowetting: from basics to applications. J. Phys.-Condens. Mat. 17(28): R705-R774 (2005) DOI: http://dx.doi.org/10.1088/0953-8984/17/28/R01

[22] M. A. Murran, and H. Najjaran: Capacitance-based droplet position estimator for digital microfluidic devices. Lab on a Chip 12(11):2053-2059 (2012) DOI: http://dx.doi.org/10.1039/C2LC21241B

[23] R. Pal, et al.: An integrated microfluidic device for influenza and other genetic analyses. Lab on a Chip 5:1024-1032 (2005) DOI: http://dx.doi.org/10.1039/B505994A

[24] M. G. Pollack, A. D. Shenderov and R. B. Fair: Electrowetting-based actuation of droplets for integrated microfluidics. Lab on a Chip 2:96-101 (2002) DOI: http://dx.doi.org/10.1039/B110474H

[25] G. J. Shah, S. Sadeghi, H. Ding, S. Chen, and R. M. van Dam: Impedance-based droplet volume and concentration measurement in digital microfluidics. MicroTAS 2011: 1308-1310. Online: http://www.rsc.org/images/LOC/2011/PDFs/Papers/438_102 3.pdf

[26] S. S. Shih, I. Barbulovic-Nad, X. Yang, R. Fobel, and A. R. Wheeler: Digital microfluidics with impedance sensing for integrated cell culture and analysis. Biosensors and bioelectronics 42:314-320 (2013) DOI: http://dx.doi.org/10.1016/j.bios.2012.10.035

[27] S. S. Shih, R. Fobel, P. Kumar, and A. R. Wheeler: A feedback control system for high-fidelity digital microfluidics. Lab on a Chip 11(3):535-540 (2011) DOI: http://dx.doi.org/10.1039/c01c00223b

[28] Y-J. Shin and J-B. Lee: Machine vision for digital microfluidics. Rev. Sci. Instrum. 81(1):014302 (2010): DOI: http://dx.doi.org/10.1063/1.3274673

[29] L. N. Soldatova, W. Aubrey, R. D. King, and A. Clare: The EXACT description of biomedical protocols. Bioinformatics 24(13): i295-i303 (2008) DOI: http://dx.doi.org/10.1093/bioinformatics/btn156

[30] I. I. Suni: Impedance methods for electrochemical sensors using nanomaterials. Trends in Anal. Chem. 27(7): 604-611 (2008) DOI: http://dx.doi.org/10.1016/j.trac.2008.03.012

[31] W. Thies, J. P. Urbanski, T. Thorsen, and S. Amarasinghe: Abstraction layers for scalable microfluidic biocomputing. Natural Computing 7(2): 255-272 (2008) DOI: http://dx.doi.org/10.1007/s11047-006-9032-6

[32] D. Woide, A. Zink, and S. Thalhammer: Technical note: PCR analysis of minimum target amount of ancient DNA. Am J Phys Anthropol, 142(2): 321-327 (2010) DOI: http://dx.doi.org/10.1002/ajpa.21268

[33] C. Zhang and D. Xing: Single-molecule DNA amplification and analysis using microfluidics. Chem Rev., 110(8): 49104947 (2010) DOI: http://dx.doi.org/10.1021/cr900081z 



\section{*Graphical Abstract}

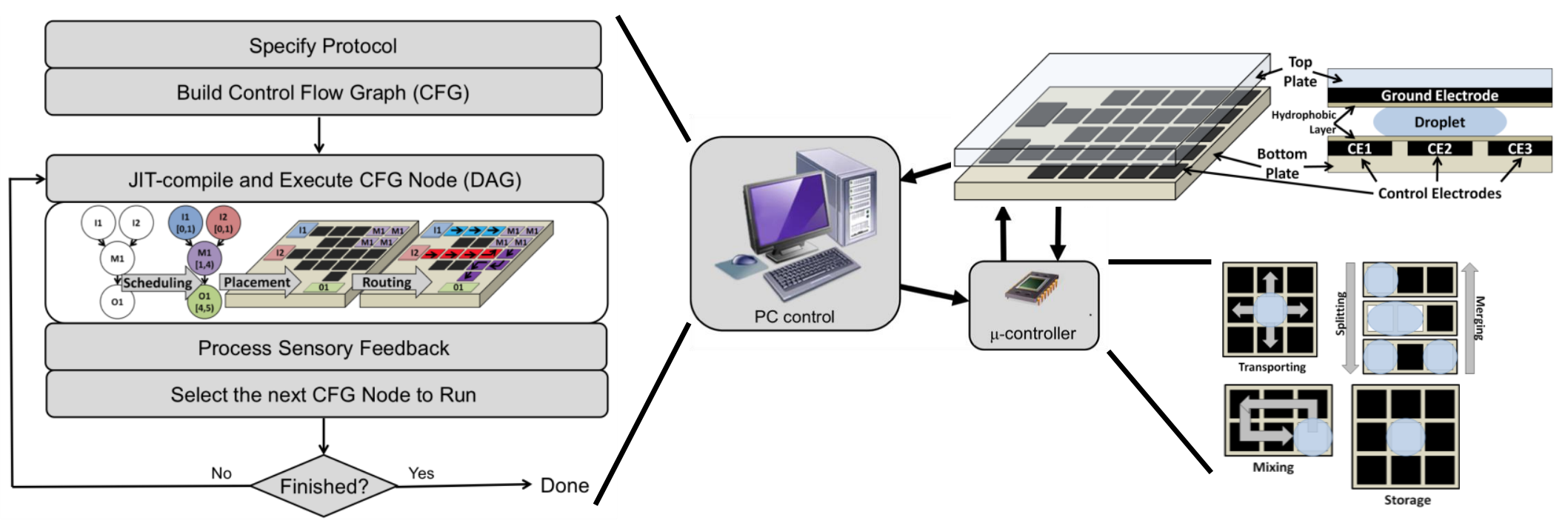

